neofilolog

Czasopismo Polskiego Towarzystwa Neofilologicznego

ISSN 1429-2173, elSSN 2545-3971, 2021, NR 56/2, 223-236

http://dx.doi.org/10.14746/n.2021.56.2.5

http://poltowneo.org/

\title{
Halina Chmiel-Bożek
}

Uniwersytet Pedagogiczny im. KEN w Krakowie https://orcid.org/ 0000-0003-4580-0485 halina.chmiel-bozek@up.krakow.pl

\section{Médiation de concepts avec la nouvelle méthode de français : C'est parti !}

\section{Mediating concepts with a new method of teaching French: C'est parti!}

The concept of mediation in foreign language teaching and learning is becoming increasingly present in literature, but due to the evolving nature of this term, many studies do not include all of its aspects. Since the publication of Common European Framework of Reference for Languages in 2001, the notion of mediation has been considerably developed and the scope of mediation activities has been extended. This article is devoted to the term "mediating concepts", which is used in Companion Volume from 2018, which updates the CEFR 2001. On the basis of the latest studies, we would like to discover what is understood by the latest idea of "mediating concepts" and what are the practical applications of these theoretical considerations, using the example of a new method of teaching French: C'est parti!

Keywords: mediation activities, mediating concepts, CEFR, C'est parti !

Słowa kluczowe: działania mediacyjne, mediacja pojęć, ESOKJ, C'est parti !

\section{Introduction}

La médiation dans l'enseignement / apprentissage des langues étrangères est de plus en plus ancrée dans la littérature (Rosen, M inns, 2008 ; De Carlo, 2012; Piccardo, 2012 ; Schädlich, 2016 ; Janowska, 2017 ; Kucharczyk, 2020), mais vu le caractère évolutif de cette notion, les travaux publiés il y a quelques années 
n'englobent pas la complexité de ce terme de manière exhaustive. Durant ces vingt dernières années, depuis la publication du Cadre européen commun de référence pour les langues : apprendre, enseigner, évaluer en 2001, la notion de médiation s'est considérablement enrichie et la portée des activités de médiation s'est élargie. C'est pourquoi, étant donné l'espace limité qu'offre un article, nous aimerions nous pencher sur une notion plus restreinte et à la fois relativement nouvelle, dont il est question dans le Cadre européen commun de référence pour les langues : apprendre, enseigner, évaluer. Volume complémentaire avec de nouveaux descripteurs publié en 2018, à savoir, la médiation de concepts, qui peut paraître vague au premier abord. Pour élucider le terme, dans un premier temps, nous allons brièvement rappeler les différentes approches de la définition de médiation, pour ensuite, sur l'exemple de la méthode de français C'est parti ! parue récemment, voir si les problèmes théoriques concernant la médiation de concepts trouvent leurs échos dans des exercices concrets proposés aux apprenants de la langue française en Pologne. L'examen critique de la méthode C'est parti ! n'est pas le but de notre travail. L'objet de l'article est d'examiner, sur la base des publications récentes, ce qui se cache derrière le nouveau terme de médiation de concepts et de voir quelles sont les possibilités pratiques de l'introduction des tâches de médiation de concepts en classe de FLE.

\section{Brève esquisse de l'évolution de la notion de médiation}

Pour saisir le sens de la notion de médiation de concepts dont il est question dans le Volume complémentaire au CECR publié en 2018, il faut revenir aux publications antérieures qui mentionnent le terme de médiation. Cette dernière n'est pas une notion nouvelle, car elle apparaît déjà dans le modèle du CECR publié en ligne en 1998 auquel se réfère Piccardo (2012 : 288). Dans cette première version, la médiation est citée parmi les autres activités langagières. Elle « constitue le prolongement de l'interaction qui à son tour participe à la fois de la réception et de la production » (Piccardo, 2012 : 288). Dans beaucoup de publications (Nort, Poccardo, 2016 : 9 ; Janowska, 2017 : 82 ; Kucharczyk, 2020 : 5), nous trouvons la reproduction du schéma ci-dessous de cette version originaire qui illustre la place de la médiation parmi les autres activités langagières:

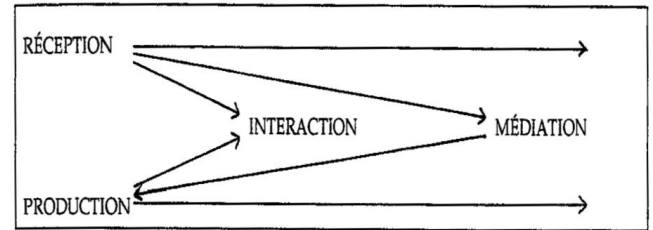

Schéma 1: Activités langagières (source : Piccardo, 2012 : 288). 
Médiation de concepts avec la nouvelle méthode de français : C'est parti !

En 2001, les auteurs du CECR ont éliminé le schéma ci-dessus mais ont quandmême conservé la définition de la notion de médiation :

Participant à la fois de la réception et de la production, les activités écrites et/ ou orales de médiation, permettent, par la traduction ou l'interprétariat, le résumé ou le compte rendu, de produire à l'intention d'un tiers une (re)formulation accessible d'un texte premier auquel ce tiers n'a pas d'abord accès direct. Les activités langagières de médiation, (re)traitant un texte déjà là, tiennent une place considérable dans le fonctionnement langagier ordinaire de nos sociétés (CECR, $2001:$ 18).

Bien que dans cette version la médiation figure à côté des autres activités langagières, les auteurs du CECR sont souvent critiqués pour ne pas la traiter de façon approfondie (M elo-Pfeifer, Schröder-Sura, $2018: 1$; Franić, 2011 : 40) et limiter sa portée à la traduction et à l'interprétariat (Piccardo, 2012 : 290 ; North, Piccardo, 2016 : 10) ou à des activités inter-linguistiques (North, Piccardo, 2016 : 11). De plus, l'absence d'échelles de descripteurs, qui pourraient permettre de décrire la progression dans les performances des apprenants, présente un autre inconvénient majeur. Ce fait est souligné par Gouiller (2019 : 100). Selon Franić, « le CECR n'a que tracé le chemin vers une découverte véritable et une description détaillée des stratégies de médiation » (2011 : 48). La médiation dans le CECR n'est donc pas suffisamment explorée, d'où la nécessité de réfléchir à cette notion, ce que suggère le titre de l'article de Piccardo publié en 2012.

La nécessité intrinsèque d'une redéfinition plus approfondie de la notion de médiation, pour combler les lacunes constatées, est à l'origine de la publication de Daniel Coste et Marisa Cavalli parue en 2015. Le concept de médiation proposé alors est beaucoup plus complexe, diversifié et inclusif. La médiation tend à « réduire l'écart entre deux pôles distants et en tension » (Coste, Cavalli, 2015 : 13). Elle vise à faciliter pour l'acteur social sa mobilité, sa perception de l'altérité, son inclusion dans la communauté. Selon cette conception, la médiation se divise en deux catégories : médiation cognitive et relationnelle. En d'autres termes, « la médiation est posée soit comme visant l'accès à des informations et connaissances et la construction de compétences (médiation cognitive), soit comme contribuant à l'interaction, à la qualité de l'échange, à la résolution de conflits (médiation relationnelle) » (Coste, Cavalli, 2015 : 13). Cette division sera reproduite dans des travaux postérieurs.

Le Cadre européen commun de référence pour les langues : apprendre, enseigner, évaluer. Élaborer des descripteurs pour illustrer les aspects de la médiation pour le CECR, publié en 2016, est entièrement consacré à la médiation. Les auteurs ont recours aux différents aspects de médiation présents dans 
les travaux antérieurs, sur les bases desquels ils distinguent quatre types de médiation : linguistique, culturelle, sociale et pédagogique (North, Piccardo, 2016 : 13-15). La conception de la médiation qu'ils proposent est quand même beaucoup plus englobante et diversifiée. En s'appuyant principalement sur les travaux de Vygotsky ${ }^{1}$ pour qui « I'activité sociale - et avec elle les différentes formes de médiation sociale et culturelle - précède l'émergence des concepts » (North, Piccardo, 2016 : 18), Brian North et Enrica Piccardo soulignent le rôle de l'interaction sociale dans le développement des connaissances.

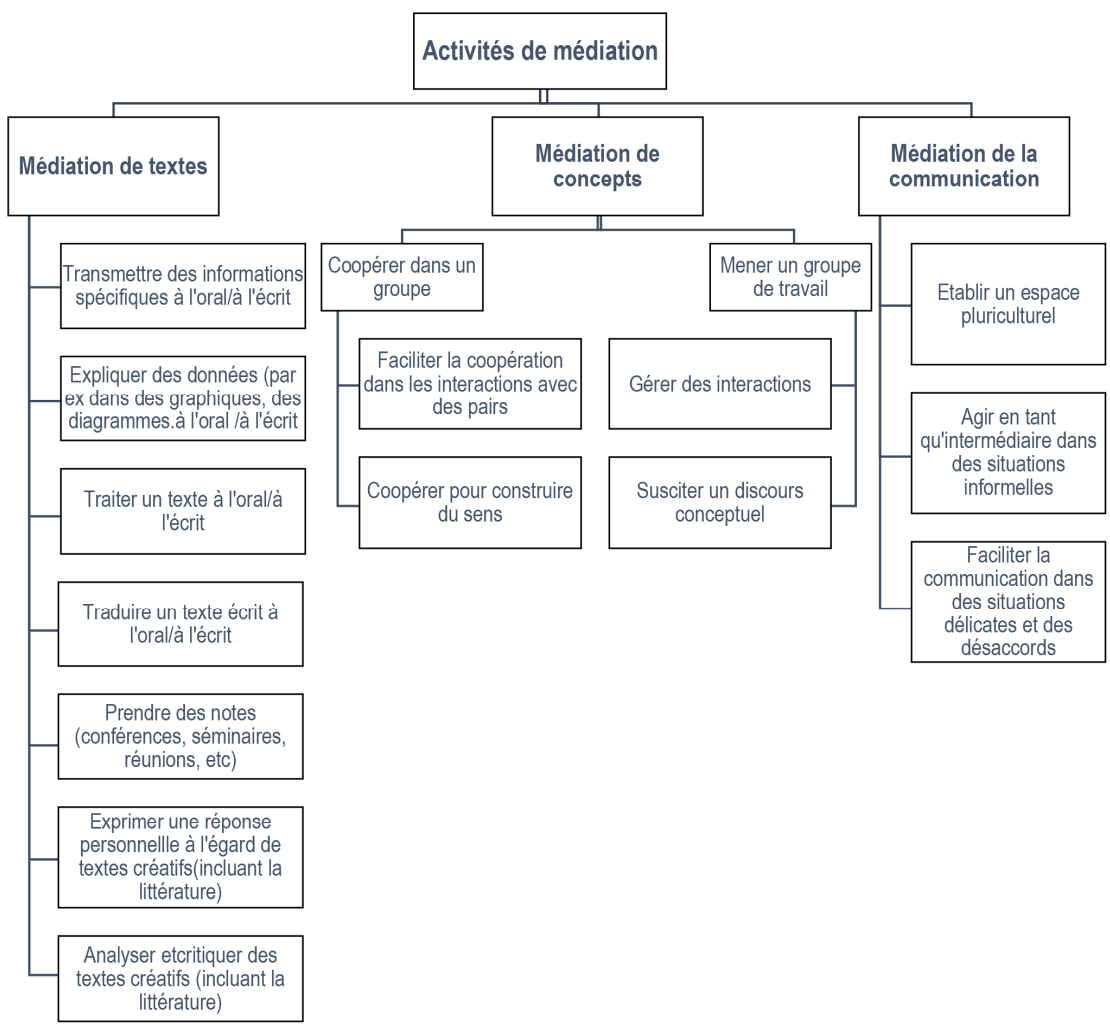

Schéma 2: Division des activités de médiation (source : CECR, 2018 : 107).

Dans le contexte éducatif, la médiation, qui est « au cœur de la co-construction du savoir » (North, Piccardo, 2016 : 18), implique donc d'aider les apprenants à s'approprier un savoir et simultanément à créer des conditions adéquates pour que cette appropriation puisse avoir lieu. Cette conception « va à

${ }^{1}$ II s'agit principalement de la publication suivante : Vigotsky, L. S. (1978). M ind in society. Cambridge: Harvard University Press. 
l'encontre de l'idée traditionnelle qu'une langue s'apprend en mémorisant des éléments linguistiques susceptibles d'être ensuite utilisés pour réaliser une activité » (North, Piccardo, 2016 : 18). Ici, à l'inverse, par le biais de la médiation on essaie de réaliser une tâche qui puisse donner du sens et structurer l'apprentissage. L'apprenant, qui participe à des conversations comme acteur social, est « mis en langage ». Pour saisir la richesse de la notion de médiation, North et Piccardo proposent la division des activités de médiation en groupes suivants : médier un texte, médier des concepts, médier la communication (North, Piccardo, 2016 : 27). La notion de médiation de concepts, qui nous intéresse dans le présent article, apparaît donc pour la première fois.

Dans le Volume complémentaire au CECR publié en 2018, les activités de médiation sont au même niveau d'importance que les autres activités langagières communicatives. La division des activités de médiation en trois groupes est reproduite et présentée dans le schéma ci-dessous. Les descripteurs élaborés facilitent la mise en pratique des activités de médiation, en permettant d'organiser un avancement dans la maîtrise de ces activités et d'en évaluer le niveau de performance.

Pour illustrer d'une manière simplifiée ces trois groupes d'activités qui créent ensemble les activités de médiation dans le contexte des autres activités langagières, Francis Gouillier, dans sa publication en 2019, a recours au schéma originel datant de 1998, en l'élargissant un peu. Comme c'est une nouveauté, nous nous permettons de le reproduire, en soulignant à la fois que la médiation de concepts, qui nous intéresse, se situe au niveau des relations.

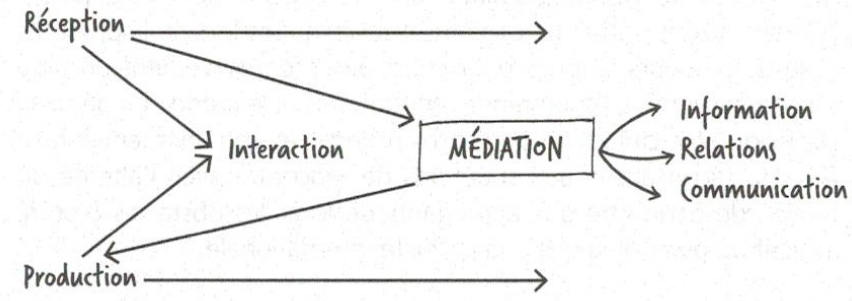

Schéma 3: Activités langagières (source : Gouiller, 2019 : 104).

\section{Médiation de concepts}

Comme nous le remarquons ci-dessus, la notion de médiation de concepts apparaît pour la première fois en 2016. Pour la définir, North et Piccardo écrivent : « on reconnaît, en éducation, que le langage est un outil utilisé pour penser à quelque chose et en parler au cours d'un processus dynamique co-constructif. Capter ce fonctionnement est donc une composante clé de l'élaboration des 
échelles de la médiation » (North, Piccardo, 2016 : 31). Selon North et Piccardo, par le langage, on peut faciliter l'accès à la connaissance et aux concepts. Ils distinguent deux voies pour y arriver : " I'une, lors d'un travail collaboratif, et l'autre quand quelqu'un a le rôle officiel ou officieux de facilitateur, de professeur ou de formateur » (North, Piccardo, 2016 : 31). Les auteurs soulignent également la nécessité de préparer les conditions adéquates pour gérer les problèmes relationnels qui s'y rapportent. Pour élucider leur conception, ils présentent deux échelles de descripteurs sous forme de tableau :

\begin{tabular}{|l|l|l|}
\hline & Établir des conditions & Développer des idées \\
\hline $\begin{array}{l}\text { Collaborer dans un } \\
\text { groupe }\end{array}$ & $\begin{array}{l}\text { Faciliter une interaction collabora- } \\
\text { tive avec des pairs }\end{array}$ & Collaborer pour construire du sens \\
\hline $\begin{array}{l}\text { Mener un groupe de } \\
\text { travail }\end{array}$ & Gérer l'interaction & Susciter un discours conceptuel \\
\hline
\end{tabular}

Tableau 1 : Échelles de descripteurs de la section « Médier des concepts » (source : North, Piccardo, 2016 : 31).

Chacune d'elles est divisée en deux éléments dont le premier concerne la mise en place des conditions pour un travail efficace (médiation relationnelle) et le second traite de l'élaboration et du développement des idées (médiation cognitive) (North, Piccardo, 2016 : 31).

En 2018, le Volume complémentaire au CECR reprend la notion de médiation de concepts. La définition proposée est presque identique à celle publiée en 2016 : « le langage sert à penser et à communiquer cette pensée dans un processus co-constructif dynamique » (CECR, 2018 : 123) et la médiation de concepts consiste à saisir ce rôle du langage. Elle a recours aux processus facilitant l'accès au savoir et aux concepts qui comprennent « deux aspects complémentaires : d'une part construire et élaborer du sens et d'autre part faciliter et encourager de bonnes conditions pour des échanges conceptuels et créatifs » (CECR, 2018 : 110). Au sens attribué par le CECR en 2018, la médiation de concepts ne diffère donc pas du modèle élaboré en 2016. Elle englobe ces activités langagières qui visent à formuler et développer efficacement des idées ou des concepts, issus d'une réflexion commune entre plusieurs interlocuteurs, et à améliorer les relations et les interactions, étant précisé que c'est le groupe qui constitue le lieu privilégié de ce type de médiation. Les échelles de descripteurs relatives à la médiation de concepts, divisées, comme dans le schéma précédent, en deux groupes, ont été présentées dans le tableau reproduit ci-dessous. Les différences, par rapport au modèle publié en 2016, semblent n'être que stylistiques. 
Médiation de concepts avec la nouvelle méthode de français : C'est parti !

\begin{tabular}{|l|l|l|}
\hline & Établir les conditions & Développer les idées \\
\hline $\begin{array}{l}\text { Coopérer dans un } \\
\text { groupe }\end{array}$ & $\begin{array}{l}\text { Faciliter la coopération dans les in- } \\
\text { teractions avec des pairs }\end{array}$ & Coopérer pour construire du sens \\
\hline $\begin{array}{l}\text { Diriger le travail de } \\
\text { groupe }\end{array}$ & Gérer les interactions & Susciter un discours conceptuel \\
\hline
\end{tabular}

Tableau 2 : Échelles de descripteurs de la section « M édiation de concepts » (source : CECR, 2018 : 123).

Comme dans le modèle antérieur, les deux échelles dans la colonne « Établir les conditions » soulignent la nécessité de créer et de maintenir des interactions positives. La médiation, à ce niveau, est un préalable nécessaire qui doit permettre, à l'étape suivante, de faciliter l'accès à de nouvelles connaissances et leur développement. Autrement dit, « chacun doit être réceptif aux points de vue des autres, de cette façon, une ambiance positive constitue souvent un prérequis pour une implication coopérative qui peut conduire à de nouvelles connaissances » (CECR, 2018 : 123).

Notons que dans les deux modèles, l'accent est mis sur l'aspect global de la communication. Les divisions marquées par les échelles de descripteurs, présentées dans les schémas ci-dessus, peuvent paraître artificielles, mais elles ne servent qu'à l'analyse et à la compréhension détaillée du processus. North et Piccardo soulignent que « les distinctions aident à la réflexion, mais une réelle communication suppose une intégration holistique des différents aspects » (North, Piccardo, 2016 : 31). Dans le Volume complémentaire au CECR, on trouve la même formulation sur les distinctions faites pour faciliter la réflexion, étant précisé que « la vraie communication demande une intégration holistique des différents aspects » (CECR, $2018: 123)$.

\section{Médier des concepts avec la méthode de français C'est parti !}

Après avoir présenté l'évolution de la notion de médiation, y compris de la médiation de concepts, d'un point de vue théorique, passons à son application pratique. Selon Radosław Kucharczyk, les exercices basés sur la médiation de concepts sont rares dans le matériel d'apprentissage des langues étrangères (Kucharczyk, 2020 : 12). L'analyse préalable des manuels de français, qui figurent sur la liste du matériel didactique autorisée par le ministère de l'Éducation nationale polonais pour les élèves de niveau secondaire ${ }^{2}$, permet de constater que les auteurs de C'est parti ! ont quand même inclus la médiation de concepts dans l'éventail des différentes activités proposées aux apprenants du français. La méthode C'est parti ! vaut la peine d'être analysée car

${ }^{2}$ Voir le site : https://podreczniki.men.gov.pl [consulté le 22 mars 2021] 
c'est une nouveauté sur le marché éditorial polonais ${ }^{3}$, rédigée uniquement par des auteurs polonais. Ses parties successives ont été publiées en 2019 et en 2020, c'est-à-dire après la publication du Volume complémentaire au CECR. Au moment de la rédaction du présent article, seules les trois premières parties sont disponibles sur le marché éditorial polonais, c'est-à-dire : C'est parti ! 1, C'est parti ! 2 et C'est parti ! 3 niveau $A 1, A 2$ et $A 2 / B 1$. D'après les informations trouvées sur le site Internet de l'éditeur ${ }^{4}$, la méthode, rédigée conformément aux dernières instructions du ministère et conçue pour les élèves des écoles secondaires en Pologne, comprendra au final quatre parties.

\subsection{Coopérer dans un groupe}

Comme indiqué dans le tableau 2 ci-dessus, " Coopérer dans un groupe » constitue la première activité majeure propre à la médiation de concepts. Elle comprend deux échelles. La première, dite relationnelle, est dédiée à « faciliter la coopération dans les interactions avec des pairs », ou en d'autres termes, à préparer les conditions propres pour un travail efficace. Selon le Volume complémentaire (CECR, 2018 : 123), nous avons affaire à ce type de médiation lorsque l'apprenant contribue au succès de la coopération au sein de son groupe, en intervenant pour orienter la discussion et en aidant à surmonter les difficultés de communication, ayant toujours à l'esprit la réalisation d'un objectif spécifique commun ou d'une tâche communicative. Dans cette situation, il n'est pas le leader dans le groupe, c'est la réussite de la coopération qui compte pour lui. Autrement dit, l'apprenant, en tant que médiateur, intervient comme facilitateur dans les échanges. De plus, les apprenants décident ensemble des étapes et des démarches à faire pour accomplir une tâche. Ils distribuent le travail dans le groupe.

La seconde échelle, dite cognitive, est destinée à « coopérer pour construire du sens ». Elle se rapporte particulièrement au travail collectif dans la résolution de problème, au brainstorming ou à la réalisation d'un projet. L'apprenant participe donc activement à la réalisation d'une tâche collective. II aide

${ }^{3}$ La liste du matériel didactique autorisée par le ministère de l'Éducation nationale polonais pour les élèves de niveau secondaire comprend 14 manuels de français dont Défi 1, En Action! 1, En Action ! 2, Exploits 1, Exploits 2, Francofolie express 1, Francofolie express 2, Texto 1 et Texto 2 étaient déjà présents sur le marché avant la publication du Volume complémentaire au CECR. Quant à \#LaClasse Al et \#LaClasse A1 H A2, l'analyse des activités de médiation de concepts incluses dans ces manuels pourrait faire l'objet d'un autre article.

${ }^{4}$ Voir le site : https:// wydawnictwodraco.pl/cest_parti_1 [consulté le 22 mars 2021] 
Médiation de concepts avec la nouvelle méthode de français : C'est parti !

les autres à trouver la solution au problème posé. Les apprenants peuvent formuler ensemble une règle, ils s'aident mutuellement pour « construire du sens ».

En réponse aux prescriptions théoriques, la méthode C'est parti ! propose cycliquement des activités qui visent à développer la coopération dans un groupe. Voici quelques exemples :

Formez les groupes de quatre personnes et écrivez un courriel à votre ami/amie marseillais(e) qui veut visiter la Pologne. Choisissez le mois de son arrivée. Décrivez le temps qu'il fait d'habitude ce mois-là et proposez des lieux à visiter. Proposez aussi des activités que vous pouvez faire pendant son séjour (C'est parti ! $1: 83$ )

Par groupes de 4 personnes, préparez par écrit une enquête sur la fréquence à laquelle chacun/chacune fait ses travaux de ménage. Puis, remplissez l'enquête et décidez qui travaille le plus à la maison (C'est parti ! $2: 65$ )

En groupe de quatre, cherchez la recette d'un plat typique français. Prenez des notes et présentez la recette à vos camarades de classe. Vous devez expliquer chaque étape de la préparation du plat (C'est parti ! 2 : 54)

En petits groupes, planifiez un voyage aux Antilles françaises. Employez le futur simple. Prévoyez le voyage et l'hébergement, les lieuxà visiter et les choses à faire. Vous vous appuierez sur le texte de l'exercice 1 et vous irez chercher des informations supplémentaires sur Internet. Vous présenterez vos plans en classe et tous ensemble vous choisirez le programme le plus intéressant (C'est parti ! $2: 97$ )

Avec ton/ta camarade de classe, partagez vos expériences liées aux jobs d'été que vous avez faits pendant les vacances. L'un(e) de vous a beaucoup apprécié ce travail. L'autre, au contraire, a trouvé cette expérience extrêmement difficile. Échangez vos expériences en utilisant le vocabulaire del'exercice précédent. Dites ce que vous avez aimé, ce que vous avez détesté et pourquoi (C'est parti ! 3 : 15)

Au niveau $A 1, A 2$ et $A 2 / B 1$ les apprenants participent à la réalisation de tâches communes simples. Ils planifient, par exemple, leur séjour aux Antilles et préparent le séjour de leur ami étranger en Pologne, réalisent une enquête concernant les travaux de ménage, présentent la recette d'un plat français ou partagent leur expérience liée au travail. Ils demandent aux collègues de leur groupe ce qu'ils en pensent, font des suggestions, demandent de répéter et/ou de reformuler tel ou tel projet si besoin est. Ils expriment leurs idées, font des propositions et des remarques à l'aide de mots très simples pour faire avancer la discussion. Le caractère coopératif du travail en groupe est souligné dans la méthode C'est parti ! par la forme de consignes où l'on utilise constamment la deuxième personne du pluriel. 


\subsection{Diriger le groupe de travail}

La seconde activité majeure propre à la médiation de concepts, définie comme « Diriger le groupe de travail », comprend également deux échelles. La première, dite relationnelle, est dédiée à la gestion des interactions en plénière et en groupes ou, autrement dit, à l'établissement des conditions propres pour un travail efficace. Dans ce cas, c'est toujours un apprenant qui « a un rôle officiel de leader dans l'organisation d'une activité communicative entre les membres d'un ou de plusieurs groupes, par exemple celui de professeur, d'animateur d'atelier, de formateur ou de président de séance » (CECR, 2018 : 126). Dans les conditions scolaires, l'apprenant est mandaté pour diriger les débats, il peut organiser le travail de la classe, inviter les participants à prendre la parole, attribuer des rôles, recueillir les différents arguments exprimés au sein du groupe. Quant à la seconde échelle, dite cognitive, définie comme " Susciter un discours conceptuel », l'apprenant en tant que médiateur peut aider d'autres personnes à formuler ou préciser leurs idées et concepts, ou même encourager à raisonner logiquement. SeIon le Volume complémentaire au CECR, « il faut pour cela fournir le cadre qui va permettre à une ou plusieurs personnes de bâtir elles-mêmes un nouveau concept, plutôt que de suivre passivement l'exemple » (CECR, 2018 : 126).

La méthode C'est parti ! propose également des activités visant à développer les compétences propres pour diriger un groupe de travail. Les exemples ci-dessous l'illustrent parfaitement :

Vérifie les compétences en mathématiques de tes camarades de classe. Propose 5 calculs de 0 à 100. Qu'ils répondent au plus vite ! (C'est parti ! $1: 23$ )

Demande à tes camarades de classe où ils vivent. Fais un questionnaire et note les résultats dans ton cahier. Dis quel est le type de logement le plus populaire dans ta classe (C'est parti ! $1: 23$ )

Décris ton appartement à tes camarades de classe. Demande de noter les informations les plus importantes. Après, vérifie s'ils ont bien noté (C'est parti ! $1: 46$ )

Choisis un état physique de l'exercice 9 et présente-le sous forme de mime. Tes camarades de classe doivent deviner de quel état il s'agit (C'est parti ! 2 : 20)

Regarde ce programme d'échange scolaire entre un établissement polonais et une école française. Certaines informations manquent. Demande à ton/ta camarade de classe ce que vont faire les élèves durant les moments du séjour pour lesquels le tableau ne prévoit aucune activité (C'est parti ! $2: 81$ )

Travaillez à deux. Demande à ton/ta collègue quel est son métier de rêve. Ensuite, formule tes conseils pour qu'il/elle puisse réussir dans ce métier, $p$. ex. Pour être mannequin, il faut que tu... (C'est parti ! $3: 51$ ) 
Au niveau $A 1, A 2$ et $A 2 / B 1$, conformément aux descripteurs propres à la médiation de concepts, l'apprenant qui joue un rôle de leader peut, par exemple, vérifier les compétences de ses camarades de classe, donner des consignes claires, aider aux formulations, affecter les tours de parole. Selon les exercices proposés par la méthode, le leader utilise des mots simples ou des expressions non verbales pour montrer son intérêt pour une idée. II aide ses camarades de classe à formuler et à préciser leurs idées et concepts concernant, par exemple, la description ou l'identification des personnes, l'endroit où l'on vit ou un programme d'échange. Les consignes sont formulées à la deuxième personne du singulier, on s'adresse donc à un apprenant qui joue le rôle de leader.

\subsection{Tâches complexes}

L'analyse détaillée des exercices proposés par la méthode C'est parti ! permet de constater que, dans beaucoup de cas, on ne peut pas classifier strictement un exercice dans tel ou tel groupe. Les tâches proposées aux apprenants sont plus complexes. II y a des exercices qui développent, de manière holistique, la coopération dans un groupe et les facultés pour diriger le travail de groupe. On ne peut pas vraiment établir de frontière fixe entre ces deux types d'activités car elle serait artificielle. II s'agit surtout d'exercices plus complexes qui comportent plusieurs étapes et visent à préparer un projet. Voici des exemples :

Formez des groupes de trois personnes. Regardez les photos avec les animaux qui appartiennent à la famille Dupont et à la famille Martin. Nommez les animaux de chaque famille. En groupes, comparez les animaux des deux photos. Utilisez les expressions de comparaison proposées dans le tableau « Exprimer la comparaison ». Sur le forum de la classe, vous pouvez organiser un concours : le groupe qui fait le plus de comparaisons gagne (C'est parti ! $2: 39$ ) ;

ou :

On va préparer en groupe un texte semblable à celui de l'exercice précédent il doit s'adresser aux touristes francophones qui viennent en Pologne, à Varsovie ou dans une autre ville. Suivez les étapes indiquées :

1. En groupe de 2 ou 3 personnes, rédigez un texte sur un des types de transports dans votre ville [...].

2. Quand lestextes sont rédigés, chaque groupe présente en classe, oralement, le moyen de transport choisi.

3. Décidez dans quel ordre vous allez joindre tous les textes présentés pour obtenir le texte général définitif.

4. Rédigez le texte général définitif, joignez tous les textes des groupes en utilisant des expressions et connecteurslogiques de l'encadré (C'est parti ! 2 : 83). 
La réalisation des exercices cités ci-dessus exige la collaboration de tous les groupes, et un apprenant au moins doit jouer le rôle de leader pour diriger le travail. Parfois, c'est l'enseignant qui joue le rôle de médiateur, comme dans l'exercice ci-dessous :

Vous allez organisez un débat sur les avantages et les inconvénients de quelques moyens de transport. L'enseignant tiendra le rôle de modérateur. Suivez les étapes:

1. Divisez-vous en groupe.

2. Chaque groupe doit choisir un moyen de transport et préparer une liste de ses avantages.

Pendant le débat, vous allez présenter ces avantages et réagir à ce que vont dire les autres groupes - qui vont parler des inconvénients du moyen de transport défendu par vous (C'est parti ! 2 : 75).

Pour terminer, notons que les activités de médiation de concepts font partie de la définition même du métier d'enseignant qui organise et dirige la communication dans la classe, s'efforce de faciliter les échanges et d'aider les apprenants à s'exprimer et à développer ensemble des idées.

\section{Conclusion}

Nous pouvons conclure que les dernières précisions du CECR concernant la médiation de concepts ont été reflétées dans les exercices proposés par les trois premières parties de la méthode de français C'est parti !. Conformément aux prescriptions théorétiques, cette nouvelle méthode propose cycliquement des activités privilégiant le travail en groupe et la réalisation de projets communs. Les apprenants sont progressivement initiés à la réalisation régulière et fréquente des activités de médiation de concepts, en commençant des plus simples jusqu'à la réalisation de projets communs plus grands pour développer la capacité de coopérer dans un groupe et de diriger le groupe de travail. L'analyse prouve que la médiation de concepts n'est pas une activité subalterne, mais qu'elle est une composante substantielle parmi les autres activités langagières. Grâce à la présence de ces activités dans C'est parti !, les exercices visant à médier des concepts seront sans doute plus souvent abordés en classes de FLE 5 .

Pour l'instant, on ne peut que regretter que, jusqu'à présent, seules les trois premières parties de la méthode aient été publiées. Dans la partie suivante

${ }^{5}$ Beaucoup de chercheurs soulignent l'impact des activités proposées par les méthodes, qui sont choisies par les enseignants, sur leurs pratiques professionnelles. En d'autres termes, les enseignants se sentent obligés de réaliser les exercices contenus dans la méthode choisie (voir : Kusiak-Pisowacka, 2015 : 67) 
Médiation de concepts avec la nouvelle méthode de français : C'est parti !

prévue, c'est-à-dire dans C'est parti ! 4, en raison du niveau de langue plus avancé, l'éventail des exercices se basant sur la médiation de concepts peut être encore plus large et diversifié.

Pour terminer, il faut garder à l'esprit que, vu la nouveauté du terme de médiation de concepts, on pourrait encore développer ou affiner des recherches concernant, par exemple, la gestion en classe de langue ou les démarches liées à la correction et à l'évaluation de tâches réalisées par des apprenants. Ce sont là des problèmes dignes d'une analyse approfondie dans un futur proche.

\section{BIBLIOGRAPHIE}

Cadre européen commun de référence pour les langues : apprendre, enseigner, évaluer. Volume complémentaire avec de nouveaux descripteurs (2018). Strasbourg: Conseil de l'Europe.

Cadre européen commun de référence pour les langues : apprendre, enseigner, évaluer. (2001). Strasbourg : Conseil de l'Europe.

Coste D., Cavalli M. (2015), Éducation, mobilité, altérité. Les fonctions de médiation de l'école. Strasbourg: Conseil de l'Europe.

De Carlo M. (2012), Traduction et médiation dans l'enseignement-apprentissage linguistique. «Études de linguistique appliquée », n0 167, pp. 299-311.

Franić I. (2011), Le concept de médiation proposé par le CECR : les représentations des apprentis traducteurs sur la médiation. « Synergies Europe », $\mathrm{n}^{\circ}$ 6, pp. 39-49.

Gouiller F. (2019), Les clés du Carde. Enjeux et actualité pour l'enseignement des langues aujourd'hui. Paris : Didier.

Janowska I. (2016), Od metody gramatyczno-tłumaczeniowej do mediacji językowej. Tłumaczenie w dydaktyce języków obcych, (in :) Lipińska E., Seretny $A$. (éds), Tłumaczenie dydaktyczne w nowoczesnym kształceniu językowym. Kraków: Księgarnia Akademicka, pp. 37-53.

Janowska I. (2017), M ediacja i działania mediacyjne w dydaktyce języków obcych. «Języki Obce w Szkole », n 3, pp. 80-86.

Kucharczyk R. (2020), Językowe działania mediacyjne na lekcjach języka obcego. Od teorii do praktyki dydaktycznej. «Języki Obce w Szkole », n² 2, pp. 5-14.

Kusiak-Pisowacka M. (2015), Ewaluacja podręcznika w nauczaniu języków obcych. « Lingwistyka stosowana », n 14, pp. 65-75.

Melo-Pfeifer S., Schröder-Sura A. (2018), Les tâches de médiation dans les manuels de Français Langue Étrangère pour le secondaire en Allemagne. « Recherches en didactique des langues et des cultures », n 15/3, pp. 1-19.

North B., Piccardo E. (2016), Cadre européen commun de référence pour les langues : apprendre, enseigner, évaluer. Élaborer des descripteurs pour 
illustrer les aspects de la médiation pour le CECR. Strasbourg : Conseil de l'Europe.

Piccardo E. (2012), M édiation et apprentissage des langues : pourquoi est-il temps de réfléchir à cette notion ? «Études de linguistique appliquée », $n^{0} 167$, pp. 285-297.

Piotrowska-Skrzypek M., Gajos M., Deckert M ., Biele D. (2019), C'est parti ! 1. Kraków: Wydawnictwo Draco Sp. z 0.0.

Piotrowska-Skrzypek M., Gajos M., Deckert M., Kalinowska E., Sowa M. (2019), C'est parti ! 2. Kraków: Wydawnictwo Draco Sp. z 0.0.

Rosen E, M inns Ph. (2008), Les activités de médiation au service du perfectionnement des compétences d'interprètes de conférences en formation illustration d'un mode de contextualisation du CECR. « Travaux de didactique du FLE », n 59 , pp. 133-151.

Schädlich B. (2016), M édiation linguistique et didactique du plurilinguisme et du pluriculturalisme, (in :) Medhat-Lecocq H., Negga D., Szende T. (éds), Traduction et apprentissage des langues. Entre médiation et remédiation. Paris : Éditions des archives contemporaines, pp. 81-91.

Sowa M., Deckert M., Piotrowska-Skrzypek M . (2020), C'est parti ! 3. Kraków: Wydawnictwo Draco Sp. z o.o.

\section{SITOGRAPHIE}

https:// podreczniki.men.gov.pl [consulté le 22 mars 2021]

https:// wydawnictwodraco.pl/cest_parti_1 [consulté le 22 mars 2021].

Received: 02.11.2020

Revised: 29.03 .2021 